\title{
Ambulatory care-sensitive conditions: their potential uses and limitations
}

\author{
Karen Hodgson, Sarah R Deeny, Adam Steventon
}

Data Analytics, The Health Foundation, London, UK

\section{Correspondence to} Dr Adam Steventon, Data Analytics, The Health Foundation, London, UK; adam.steventon@health.org.uk

Accepted 8 February 2019 Published Online First 28 February 2019

\section{SLinked}

- http://dx.doi.org/10.1136/ bmjgs-2018-008002

\section{Check for updates}

(c) Author(s) (or their employer(s)) 2019. No commercial re-use. See rights and permissions. Published by BMJ.

To cite: Hodgson $\mathrm{K}$, Deeny SR, Steventon A. BMJ Qual Saf

2019;28:429-433.
Emergency admission rates for ambulatory care-sensitive conditions (ACSCs) have been used by both researchers and policy makers as an indicator to assess healthcare systems. ${ }^{1-3}$ ACSCs are a set of conditions such as asthma and diabetes, where the need for emergency admissions is thought to be avoidable. ${ }^{45}$ They are designed to capture how ambulatory care might impact on rates of emergency admissions. However, in this issue of $B M J$ Quality and Safety, Lynch et $a l^{6}$ question the merits of distinguishing ACSCs from other emergency admissions. Examining factors associated with county-level variation in emergency admissions in Ireland, they found similar factors were associated with both all emergency admissions and ACSCs. Lynch and colleagues concluded that 'the distinction between potentially avoidable and all other emergency admissions may not be as useful as previously believed'. 6

Here, we discuss the value of using ACSCs. We argue that they give important information on potentially avoidable demand for healthcare, but can only be used as an effective indicator after accounting for the drivers of demand for care that are common to all hospital admissions. Further, to reduce potentially avoidable emergency admissions, we must understand the mechanisms which link population factors such as deprivation to higher ACSC admission rates. In addition to understanding demand, ACSCs can provide indirect insights into the quality of ambulatory care provided, although it is important to recognise that they do not capture all facets of high quality care.

\section{THE IMPORTANCE OF REDUCING EMERGENCY ADMISSIONS}

Policy makers generally seek to reduce emergency hospital admission rates (ie, where a patient is admitted to hospital urgently and the admission is unplanned). Emergency admissions to hospitals carry risks to patients; for vulnerable patients, even short admissions can lead to infection, loss of mobility or increased frailty from inactivity. If treatment is unavoidable, many patients would prefer to be treated closer to home. Emergency admissions are also expensive, costing the NHS $£ 18.0$ billion in $2017 / 2018,^{7}$ and they can impact on the ability of hospitals to deliver elective care. In the English NHS, emergency patients occupy nearly three out of four hospital beds at any one point in time. ${ }^{8}$ Emergency admissions are unpredictable, so when hospitals are operating at high rates of bed occupancy (as in England), sudden increases in emergency admissions result in cancelled elective operations. ${ }^{9}$

Despite the undesirability of emergency admissions, in many healthcare systems rates are nevertheless rising quickly, with associated increases in costs. For example, in England emergency admissions increased by $42 \%$ between 2006 and 2017; the total cost of these also grew by $£ 5.5$ bn in the period from 2006 to $2016 .{ }^{8}$ To reverse this trend of increasing emergency admissions, metrics are key for the successful development and evaluation of improvement strategies.

\section{THE DEVELOPMENT OF ACSCS}

Monitoring rates of emergency admissions gives insight into the overall demand in the emergency care system. However, many emergency admissions are medically necessary and unavoidable. To understand how emergency admission rates could be reduced, it may be more useful to focus on admissions that could have been avoided. ACSCs specifically focus on emergency admissions that 
are potentially avoidable with optimum ambulatory care. This might be with better management of acute episodes outside of hospital. For example, an acute asthma episode might be rapidly dealt with by a GP without requiring an emergency admission. In other cases, preventing exacerbations of chronic illnesses may avoid the need for emergency care altogether, for example, via improved primary care management of a patient's diabetes.

To identify admissions that are potentially avoidable at a patient level, individual case notes can be assessed. But, within large healthcare datasets, a more easily derived proxy is to calculate the rate of admissions for conditions where there is known to be a high proportion of avoidable cases. Expert consensus has been used to group conditions into lists based on avoidability. ${ }^{4} 10$ ACSCs are conditions where ambulatory care is deemed to have a large potential to reduce the need for emergency admission. On the other side of the spectrum, there are 'care-insensitive' conditions, where prior care is thought to be largely irrelevant (eg, acute appendicitis). ${ }^{5}$ ACSCs are an indicator, not a direct measure of ambulatory care, so there may be non-care related factors which contribute to admission rates. But with careful use, we can use ACSCs to observe variation within a healthcare system and identify areas for further investigation, where demand for healthcare might be better managed with improvements to ambulatory care.

\section{ACSCS AS A MONITORING TOOL}

Admission rates for ACSCs have been used as a tool for monitoring healthcare systems in multiple countries, including the USA, ${ }^{2}$ England ${ }^{11}$ and Australia. ${ }^{12}$ Table 1 outlines the conditions included as ACSCs in the NHS Outcomes Framework; these make up approximately $14 \%$ of emergency admissions in England. ${ }^{8}$ In recent years, there has also been growing interest developing the equivalent of ACSC metrics to assess particular parts of the healthcare system. For example, a set of 'urgent care sensitive' conditions has been developed to reflect the influence of the urgent-care system (including primary care, ambulance services and hospital admission protocols) on emergency admissions. ${ }^{10}$ There are also programmes focusing on conditions where emergency admissions are potentially avoidable among care-home residents. ${ }^{13} 14$

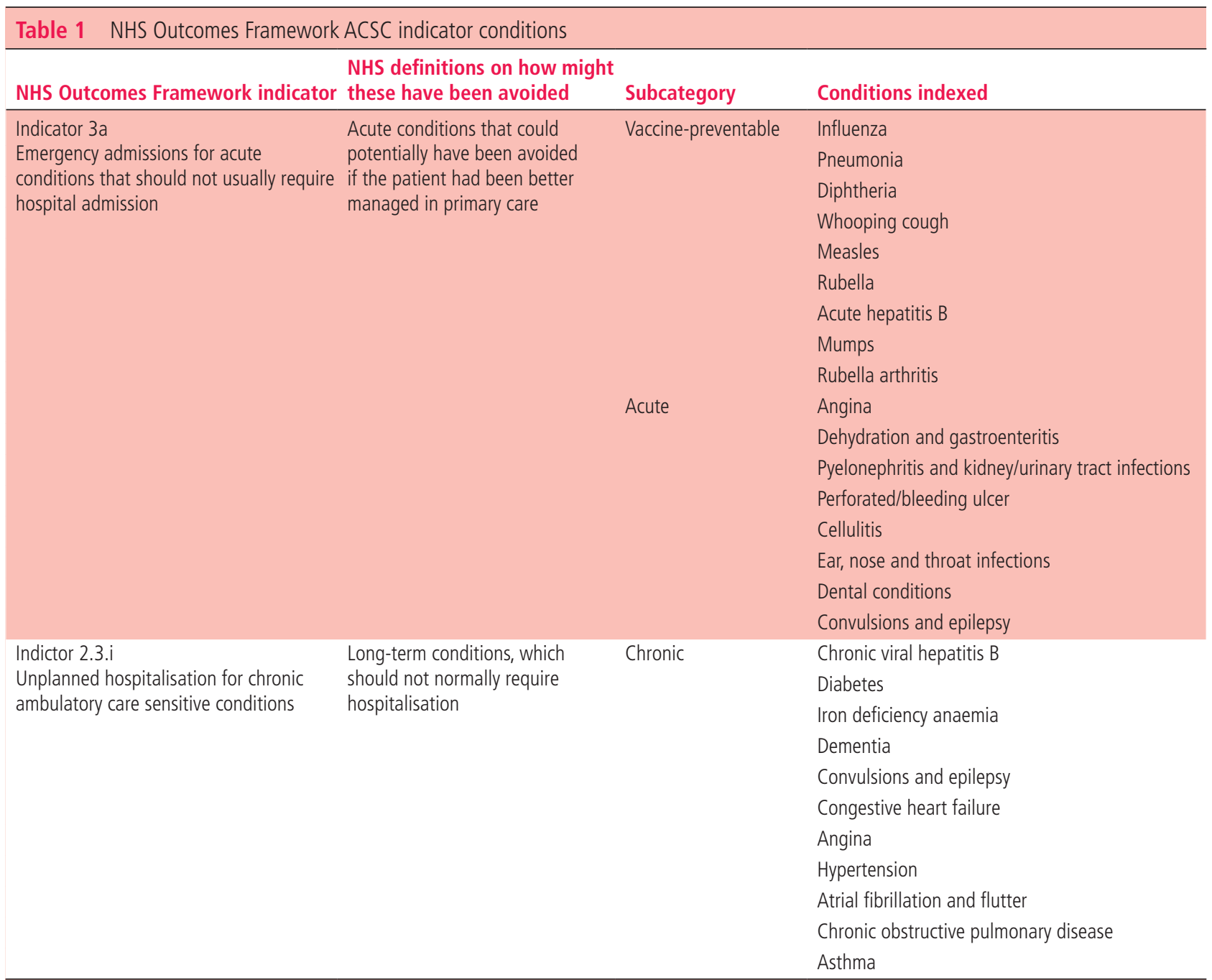




\section{THE IMPORTANCE OF CONTEXT}

One concern as ACSCs become more widely used is that there is a risk that they are used as a 'black box' indicator of ambulatory care, without considering alignment between the context in which lists were developed and the context in which they are being applied. In many cases, lists have been adapted from the USA and do not always reflect the mix of conditions and availability of services in other healthcare systems, particularly when applied to healthcare systems with universal access to care. For example, Purdy et $a l^{15}$ highlight the very low incidence of gangrene within England, limiting its relevance as an indicator of ambulatory care. These context-specific influences need to be carefully considered when using ACSC indicators.

\section{THE MULTIFACTORIAL NATURE OF ALL HOSPITAL ADMISSIONS}

Despite their use to monitor healthcare systems, the effectiveness of ACSCs as an indicator has been debated. ${ }^{15}{ }^{16}$ Recent work from Lynch et al ${ }^{6}$ concluded that 'the distinction between potentially avoidable and all other emergency admissions may not be as useful as previously believed when attempting to identify the causes of regional variation in emergency admission rates', when looking at county-level variation in emergency admissions in Ireland from 2014 to 2016. The researchers found that similar factors (unemployment, hospital admission policy and private health insurance coverage) were associated with both care-sensitive and total emergency admissions. However, we would argue that this simply captures the drivers of demand that are common across all emergency admissions. For ACSCs to fulfil their purpose in tapping into potentially avoidable admissions, an approach which accounts for these general drivers of all admissions is needed. Indeed, within the appendix, Lynch et al examined the ratio of care-sensitive to total admissions, finding that older, unemployed and urban populations show higher rates of admissions specifically for care-sensitive conditions.

\section{MECHANISMS WHICH DRIVE VARIATION IN ACSCS}

Potentially avoidable admissions are consistently associated with population factors such as deprivation. ${ }^{17-19}$ Indeed, one of the first uses of ACSCs from Billings et $a l^{4}$ examined variation in emergency admissions in New York City and found low-income areas had much higher admissions rates for ACSCs as compared with high-income areas, with no corresponding increase in care-insensitive admission rates. But observing an association between potentially avoidable admissions and deprivation does not reveal the mechanism driving this; more detailed evaluation is needed.

This requires access to more fine-grained data on specific aspects of ambulatory care. For example, some studies have examined the possible impact of continuity of care ${ }^{20}$ managed care plans ${ }^{21}$ or pay for performance schemes ${ }^{22}$ on ACSC rates. The aim of this work is to build evidence-based approaches to reduce potentially avoidable admissions. But given the complex nature of healthcare outcomes, this is difficult to achieve. Furthermore, because there are a diverse number of conditions included under the banner of ACSCs, methods which effectively reduce admission rates for all ACSCs will be broad efforts to improve ambulatory care for a cross-section of patients, rather than targeted, disease-specific actions. Our understanding of what works and what does not work in this area continues to evolve (eg, see Ref. 17 for a review), and in many cases, the efficacy of an approach may be specific to particular healthcare contexts.

Even with apparently equal ambulatory care, there may still be underlying factors driving variation in ACSCs. For instance, sociocultural norms or increased need might mean that some groups receive a lower standard of care or are more vulnerable to the quality of care received. ${ }^{23}$ Targeting of ambulatory care for these groups may help reduce demand on the emergency care system. Alternatively, it might be that higher rates of potentially avoidable admissions for some groups are driven by non-healthcare factors such as community, employment and housing. ${ }^{24}$ These drivers of emergency admissions may be beyond the scope of clinicians, but still impact on the healthcare system, so are of high relevance to policy makers looking to understand and manage demand for emergency admissions. These broader influences on healthcare needs contribute to the complexity of interpreting ACSCs as an indicator.

\section{DIFFICULTIES IN DRAWING CONCLUSIONS ABOUT QUALITY OF CARE}

Beyond understanding where demand might be better managed using ambulatory care, ACSCs might be used to infer the quality of ambulatory care received. Despite its importance, quality is notoriously complex to assess. Many health datasets focus on how often patients use services (eg, emergency admission rates) and contain relatively little information on clinical outcomes and care processes, and less information still on other aspects of care quality like patient-centeredness. ${ }^{25}$ ACSCs allow us to explore variation in care using hospital data which is routinely collected in most healthcare systems and relatively easy to access. However, even if the mechanism driving ACSC admission rates is related to the provision of care, it is important to bear in mind the limited window ACSCs give us into quality of care. When considering the components of high quality care, the Institute of Medicine identified six 'Aims for Improvement'; that healthcare should be safe, effective, efficient, timely, patient-centred and equitable. ${ }^{26}$ Admission rates for ACSCs capture indirect evidence relating to some of these aims, but do not capture the full picture of quality, missing key elements including patient-centeredness. Therefore, it may be 
one of the few available proxy measures of care quality but it is difficult to conclude by looking at emergency admission rates alone whether each of the aspects of quality of care have necessarily improved.

\section{CONCLUSION}

In the 25 years since their first description, ${ }^{4}$ ACSCs have been applied to an increasing number of different contexts. As a result, our understanding of this indicator has shifted over time. Emergency admission rates remain an important outcome to measure, with implications for both healthcare managers and patients. Focusing specifically at admission rates for ACSCs can be a valuable indicator of demand for care, but appropriate controls accounting for the multifactorial drivers of all hospital admissions are needed.

We argue that as an indicator, ACSCs are useful for understanding demand in the emergency system and give an insight into the quality of ambulatory care (although limited). But often the literature focuses on testing for associations between ACSC rates and population factors (such as deprivation). These associations are only a small part of the story-questions need to be asked regarding the mechanisms by which these population factors are linked to increased emergency admission rates, examining the impact of specific aspects of ambulatory care provision.

Ultimately, reliance on ACSCs as an indicator is driven by the lack of alternative sources of data that effectively captures the impact of ambulatory care. This points to a need for investment in other datasets with greater focus on care processes (eg, clinical audits and registries, see Ref. 27). Until then, rates of emergency admissions for ACSCs may remain the best available data to researchers seeking to understand the relationship between emergency outcomes for patients and the care that they received.

Funding The authors have not declared a specific grant for this research from any funding agency in the public, commercial or not-for-profit sectors.

Competing interests The authors work in the Data Analytics Division at the Health Foundation, which co-owns BMJ Quality \& Safety.

Patient consent for publication Not required.

Provenance and peer review Commissioned; internally peer reviewed.

\section{REFERENCES}

1 Busby J, Purdy S, Hollingworth W. A systematic review of the magnitude and cause of geographic variation in unplanned hospital admission rates and length of stay for ambulatory care sensitive conditions. BMC Health Serv Res 2015;15.

2 AHRQ Quality Indicators. Guide to prevention quality indicators: hospital admission for ambulatory care sensitive conditions. AHRQ Pub No. 02-R0203. Rockville, MD: Agency for Healthcare Research and Quality, 2001.

3 NHS England. Emergency admissions for ambulatory care sensitive conditions - characteristics and trends at national level, 2014.
4 Billings J, Zeitel L, Lukomnik J, et al. Impact of socioeconomic status on hospital use in New York City. Health Aff 1993;12:162-73.

5 Sanderson C, Dixon J. Conditions for which onset or hospital admission is potentially preventable by timely and effective ambulatory care. J Health Serv Res Policy 2000;5:222-30.

6 Lynch B, Fitzgerald AP, Corcoran P, et al. Drivers of potentially avoidable emergency admissions in Ireland: an ecological analysis. BMJ Qual Saf 2019;28:438-49.

7 NHS Improvement. Reference costs 2017/18: highlights, analysis and Introduction to the data, 2018. Available: https:// improvement.nhs.uk/documents/1972/1_-_Reference_costs_ 201718.pdf

8 Steventon A, Deeny S, Friebel R. Emergency hospital admissions in England: which may be avoidable and how? The Health Foundation; 2018: 1-21. https://www.health. org.uk/publications/emergency-hospital-admissions-inengland-which-may-be-avoidable-and-how. 978-1-91161511-8.

9 NHS England. Operational update from the NHS national emergency pressures panel, 2018. Available: https://www. england.nhs.uk/2018/01/operational-update-from-the-nhsnational-emergency-pressures-panel/

10 Nicholl J, Coleman P, Jenkins J, et al. MCRU programme 2006-2010 emergency and urgent care systems final report of phase, 2011. Available: https://www.sheffield.ac.uk/scharr/ sections/hsr/mcru/reports

11 Health and Social Care Information Centre. NHS outcomes framework. Hscic 2016:4-49.

12 Falster M, Jorm L. A guide to the potentially preventable hospitalisations indicator in Australia, 2017. Available: www. safetyandquality.gov.au [Accessed 18 Dec 2018].

13 Care Quality Commission. The state of health care and adult social care in England in 2012/2013, 2013. Available: https:// assets.publishing.service.gov.uk/government/uploads/system/ uploads/attachment_data/file/264035/0838.pdf

14 Ouslander JG, Bonner A, Herndon L, et al. The interventions to reduce acute care transfers (interact) quality improvement program: an overview for medical directors and primary care clinicians in long term care. Journal of the American Medical Directors Association 2014;15:162-70.

15 Purdy S, Griffin T, Salisbury C, et al. Ambulatory care sensitive conditions: terminology and disease coding need to be more specific to aid policy makers and clinicians. Public Health 2009;123:169-73.

16 Longman JM, Passey ME, Ewald DP, et al. Admissions for chronic ambulatory care sensitive conditions - a useful measure of potentially preventable admission? BMC Health Serv Res 2015;15:1-4.

17 Purdy S. Avoiding hospital admissions what does the research evidence say? London The King's Fund; 2010. https://www. kingsfund.org.uk/publications/avoiding-hospital-admissions

18 Purdy S, Huntley A. Predicting and preventing avoidable hospital admissions. The Journal of the Royal College of Physicians of Edinburgh2013;43:340-4.

19 O'Cathain A, Knowles E, Maheswaran R, et al. A system-wide approach to explaining variation in potentially avoidable emergency admissions: national ecological study. BMJ Qual Saf 2014;23:47-55.

20 Barker I, Steventon A, Deeny SR. Association between continuity of care in general practice and hospital admissions for ambulatory care sensitive conditions: cross sectional study of routinely collected, person level data. BMJ 2017;356. 
21 Bindman AB, Chattopadhyay A, Osmond DH, et al. The impact of Medicaid managed care on hospitalizations for ambulatory care sensitive conditions. Health Serv Res $2005 ; 40: 19-38$.

22 Harrison MJ, Dusheiko M, Sutton M, et al. Effect of a national primary care pay for performance scheme on emergency hospital admissions for ambulatory care sensitive conditions: controlled longitudinal study. BMJ 2014;349:g6423-8.

23 Tudor Hart J. The inverse care law. The Lancet 1971;297:405-12.
24 Lovell N, Bibby J. What makes us healthy? An introduction to the social determinants of health. London: The Health Foundation, 2018.

25 Deeny SR, Steventon A. Making sense of the shadows: priorities for creating a learning healthcare system based on routinely collected data. BMJ Qual Saf 2015;24:505-15.

26 Institute of Medicine. Crossing the quality chasm: a new health system for the 21st century. National Academy Press, 2001.

27 Redfern-Tofts D. Unlocking the power of audits and registries, 2018. The Health Foundation. Available: https://www.health. org.uk/blogs/insight-research-programme-unlocking-thepower-of-audits-and-registries 